\title{
On the Capacity Achieving Covariance Matrix for Frequency Selective MIMO Channels Using the Asymptotic Approach
}

\author{
Florian Dupuy \\ Thales Communication EDS/SPM \\ 160 Bd de Valmy \\ 92704 Colombes Cedex, France
}

\author{
Philippe Loubaton \\ Université de Paris-Est \\ IGM LabInfo, UMR-CNRS 8049 \\ 5, Bd Descartes, Champs-sur-Marne \\ 77454 Marne-la-Vallée Cedex 2, France
}

\begin{abstract}
In this contribution, an algorithm for evaluating the capacity-achieving input covariance matrices for frequency selective Rayleigh MIMO channels is proposed. In contrast with the flat fading Rayleigh cases, no closed-form expressions for the eigenvectors of the optimum input covariance matrix are available. Classically, both the eigenvectors and eigenvalues are computed numerically and the corresponding optimization algorithms remain computationally very demanding.

In this paper, it is proposed to optimize (w.r.t. the input covariance matrix) a large system approximation of the average mutual information derived by Moustakas and Simon. An algorithm based on an iterative water filling scheme is proposed, and its convergence is studied. Numerical simulation results show that, even for a moderate number of transmit and receive antennas, the new approach provides the same results as direct maximization approaches of the average mutual information.
\end{abstract}

\section{INTRODUCTION}

When the channel state information is available at both the receiver and the transmitter of a MIMO system, the problem of designing the transmitter in order to maximize the (Gaussian) mutual information of the system has been addressed successfully in a number of papers. This problem is however more difficult when the transmitter has the knowledge of the statistical properties of the channel, a more realistic assumption in the context of mobile systems. In this case, the mutual information is replaced by the average mutual information (EMI), which, of course, is more complicated to optimize.

The optimization problem of the EMI has been addressed extensively in the case of certain flat fading Rayleigh channels. In the context of the so-called Kronecker model, it has been shown by various authors (see e.g. [1] for a review) that the eigenvectors of the optimal input covariance matrix must coincide with the eigenvectors of the transmit correlation matrix. It is therefore sufficient to evaluate the eigenvalues of the optimal matrix, a problem which can be solved by using standard optimization algorithms. Similar results have been obtained for flat fading uncorrelated Rician channels ([2]).

In this paper, we consider this EMI maximization problem in the case of popular frequency selective MIMO channels (see e.g. [3], [4]) with independent paths. In this context, the eigenvectors of the optimum transmit covariance matrix have no closed expressions, so that both the eigenvalues and the eigenvectors of the matrix have to be evaluated numerically. For this, it is possible to adapt the approach of [5] developed in the context of correlated Rician channels. However, the corresponding algorithms are computationally very demanding as they heavily rely on intensive Monte-Carlo simulations. We therefore propose to optimize the approximation of the EMI, derived by Moustakas and Simon ([4]), in principle valid when the number of transmit and receive antennas converge to infinity at the same rate, but accurate for realistic numbers of antennas. This will turn out to be a simpler problem. We mention that, while [4] contains some results related to the structure of the argument of the maximum of the EMI approximation, [4] does not propose any optimization algorithm.

We first review the results of [4] related to the large system approximation of the EMI. The expression of the approximation depends on the solutions of a non linear system. The existence and the uniqueness of the solutions is not addressed in [4]. As our optimization algorithm needs to solve this system, we clarify this crucial point. Next, we present our maximization algorithm of the EMI approximation. It is based on an iterative waterfilling algorithm which, in some sense, can be seen as a generalization of [6] devoted to the Rayleigh context and of [7] devoted to the correlated Rician case: each iteration will be devoted to solve the above mentioned system of nonlinear equations as well as a standard waterfilling problem. It is proved that the algorithm converges towards the optimum input covariance matrix as long as it converges. 1 .

The paper is organized as follows. Section III is devoted to the presentation of the channel model, the underlying assumptions, the problem statement. The maximization problem of the EMI approximation is studied in section III Numerical results are provided in section IV

\footnotetext{
${ }^{1}$ Note however that we have been unable to prove formally its convergence.
} 


\section{Problem Statement}

\section{A. General Notations}

In this paper, the notations $s, \mathbf{x}, \mathbf{M}$, stand for scalars, vectors and matrices, respectively. As usual, $\|\mathbf{x}\|$ represents the Euclidian norm of vector $\mathbf{x}$, and $\|\mathbf{M}\|, \rho(\mathbf{M})$ and $|\mathbf{M}|$ respectively stand for the spectral norm, the spectral radius and the determinant of matrix $\mathbf{M}$. The superscripts $(.)^{T}$ and $(.)^{H}$ represent respectively the transpose and transpose conjugate. The trace of $\mathbf{M}$ is denoted by $\operatorname{Tr}(\mathbf{M})$. The mathematical expectation operator is denoted by $\mathbb{E}(\cdot)$

All along this paper, $r$ and $t$ stand for the number of receive and transmit antennas. Certain quantities will be studied in the asymptotic regime $t \rightarrow \infty, r \rightarrow \infty$ in such a way that $t / r \rightarrow$ $c \in(0, \infty)$. In order to simplify the notations, $t \rightarrow \infty$ should be understood from now on as $t \rightarrow \infty, r \rightarrow \infty$ and $t / r \rightarrow$ $c \in(0, \infty)$.

Several variables used throughout this paper depend on various parameters, e.g. the number of antennas, the noise level, the covariance matrix of the transmitter, etc. In order to simplify the notations, we may not always mention all these dependencies.

\section{B. Channel model}

We consider a wireless MIMO link with $t$ transmit and $r$ receive antennas corrupted by a multi-paths propagation channel. The discrete-time propagation channel between the transmitter and the receiver is characterized by the input-output equation

$$
\mathbf{y}(n)=\sum_{l=1}^{L} \mathbf{H}_{l} \mathbf{s}(n-l+1)+\mathbf{n}(n)=[\mathbf{H}(z)] \mathbf{s}(n)+\mathbf{n}(n)
$$

where $\mathbf{s}(n)=\left(s_{1}(n), \ldots, s_{t}(n)\right)^{T}$ represents the transmit vector at time $n, \mathbf{y}(n)=\left(y_{1}(n), \ldots, y_{r}(n)\right)^{T}$ the receive vector, and where $\mathbf{n}(n)$ is an additive Gaussian noise such that $\mathbb{E}\left(\mathbf{n}(n) \mathbf{n}(n)^{H}\right)=\sigma^{2} \mathbf{I}$. $\mathbf{H}(z)$ denotes the transfer function of the discrete-time equivalent channel defined by

$$
\mathbf{H}(z)=\sum_{l=1}^{L} \mathbf{H}_{l} z^{-(l-1)}
$$

Each coefficient $\mathbf{H}_{l}$ is assumed to be a Gaussian random matrix given by

$$
\mathbf{H}_{l}=\frac{1}{\sqrt{t}}\left(\mathbf{C}^{(l)}\right)^{1 / 2} \mathbf{W}_{l}\left(\tilde{\mathbf{C}}^{(l)}\right)^{1 / 2}
$$

where $\mathbf{W}_{l}$ is a $r \times t$ random matrix whose entries are independent and identically distributed complex circular Gaussian random variables, with zero mean and unit variance. The matrices $\mathbf{C}^{(l)}$ and $\tilde{\mathbf{C}}^{(l)}$ are positive definite, and account for the receive and transmit antenna correlation. We also assume that for each $k \neq l$, matrices $\mathbf{H}_{k}$ and $\mathbf{H}_{l}$ are independent.

In the context of this paper, the channel matrices are assumed perfectly known at the receiver side. However, only the statistics of the $\left(\mathbf{H}_{l}\right)_{l=1, \ldots, L}$, i.e. matrices $\left(\tilde{\mathbf{C}}^{(l)}, \mathbf{C}^{(l)}\right)_{l=1, \ldots, L}$, are available at the transmitter side.

\section{Ergodic capacity of the channel.}

Let $\mathbf{Q}\left(e^{2 i \pi \nu}\right)$ be the $t \times t$ spectral density matrix of the transmit signal $\mathbf{s}(n)$, which is assumed to verify the transmit power condition

$$
\frac{1}{t} \int_{0}^{1} \operatorname{Tr}\left(\mathbf{Q}\left(e^{2 i \pi \nu}\right)\right) d \nu=1
$$

Then, the (Gaussian) ergodic mutual information $I(\mathbf{Q}()$. between the transmitter and the receiver is defined as

$$
I(\mathbf{Q}(.))=\mathbb{E}_{\mathcal{W}}\left[\int_{0}^{1} \log \left|\mathbf{I}_{r}+\frac{1}{\sigma^{2}} \mathbf{H}(.) \mathbf{Q}(.) \mathbf{H}(.)^{H}\right| d \nu\right]
$$

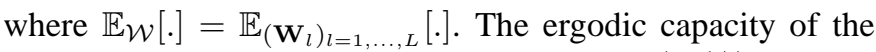
MIMO channel is equal to the maximum of $I(\mathbf{Q}()$.$) over the$ set of all spectral density matrices satisfying the constraint (4). The hypotheses formulated on the statistics of the channel allow however to limit the optimization to the set of positive matrices which are independent of the frequency $\nu$. This is because the probability distribution of matrix $\mathbf{H}\left(e^{2 i \pi \nu}\right)$ is clearly independent of the frequency $\nu$. More precisely, the mutual information $\mathbf{I}(\mathbf{Q}()$.$) is also given by$

$$
I(\mathbf{Q}(.))=\mathbb{E}_{\mathbf{H}}\left[\int_{0}^{1} \log \left|\mathbf{I}_{r}+\frac{1}{\sigma^{2}} \mathbf{H}(1) \mathbf{Q}(.) \mathbf{H}(1)^{H}\right| d \nu\right]
$$

where $\mathbf{H}=\sum_{l=1}^{L} \mathbf{H}_{l}=\mathbf{H}(1)$. Using the concavity of the logarithm, we obtain that

$$
I(\mathbf{Q}(.)) \leq \mathbb{E}_{\mathbf{H}}\left[\log \left|\mathbf{I}_{r}+\frac{1}{\sigma^{2}} \mathbf{H}(1)\left(\int_{0}^{1} \mathbf{Q}(.) d \nu\right) \mathbf{H}(1)^{H}\right|\right]
$$

We denote by $\mathcal{C}$ the cone of non negative hermitian matrices, and by $\mathcal{C}_{1}$ the subset of all matrices $\mathrm{Q}$ of $\mathcal{C}$ satisfying $\frac{1}{t} \operatorname{Tr}(\mathbf{Q})=1$. If $\mathbf{Q}$ is an element of $\mathcal{C}_{1}$, the mutual information $I(\mathbf{Q})$ reduces to

$$
I(\mathbf{Q})=\mathbb{E}_{\mathbf{H}}\left[\log \left|\mathbf{I}_{r}+\frac{1}{\sigma^{2}} \mathbf{H} \mathbf{Q} \mathbf{H}^{H}\right|\right]
$$

It is strictly concave on the convex set $\mathcal{C}_{1}$ and reaches its maximum at a unique element $\mathbf{Q}_{*} \in \mathcal{C}_{1}$. It is clear that if $\mathbf{Q}\left(e^{2 i \pi \nu}\right)$ is any spectral density satisfying (4), then the matrix $\int_{0}^{1} \mathbf{Q}\left(e^{2 i \pi \nu}\right) d \nu$ is an element of $\mathcal{C}_{1}$. Therefore,

$$
I(\mathbf{Q}(.)) \leq I\left(\mathbf{Q}_{*}\right)
$$

for each spectral density matrix verifying (4). This shows that the maximum of function $I$ over the set of all spectral densities satisfying (4) is reached on the set $\mathcal{C}_{1}$. The ergodic capacity $\mathcal{C}_{E}$ of the channel is thus equal to

$$
\mathcal{C}_{E}=\max _{\mathbf{Q} \in \mathcal{C}_{1}} I(\mathbf{Q})
$$

If the matrices $\left(\mathbf{C}^{(l)}\right)_{l=1, \ldots, L}$ coincide with a matrix $\mathbf{C}$, matrix $\mathbf{H}$ follows a Kronecker model with transmit and receive covariance matrices $\sum_{l=1}^{L} \tilde{\mathbf{C}}^{(l)}$ and $\mathbf{C}$ respectively [8]. In this case, the eigenvectors of the optimum matrix $\mathbf{Q}_{*}$ coincide with the eigenvectors of $\sum_{l=1}^{L} \tilde{\mathbf{C}}^{(l)}$. The situation is similar if the transmit covariance matrices $\left(\tilde{\mathbf{C}}^{(l)}\right)_{l=1, \ldots, L}$ coincide. In the most general case, the eigenvectors of $\mathbf{Q}_{*}$ have however no 


$$
\bar{I}(\mathbf{Q})=\log \left|\mathbf{I}+\sum_{l=1}^{L} \tilde{\delta}_{l}(\mathbf{Q}) \mathbf{C}^{(l)}\right|+\log \left|\mathbf{I}+\mathbf{Q}\left(\sum_{l=1}^{L} \delta_{l}(\mathbf{Q}) \tilde{\mathbf{C}}^{(l)}\right)\right|-\sigma^{2} t\left(\sum_{l=1}^{L} \delta_{l}(\mathbf{Q}) \tilde{\delta}_{l}(\mathbf{Q})\right)
$$

closed form expression. The evaluation of $\mathbf{Q}_{*}$ and of the channel capacity $\mathcal{C}_{E}$ is thus a more difficult problem. A possible solution consists in adapating the Vu-Paulraj approach ([5]) to the present context. However, the algorithm presented in [5] is very demanding since the evaluation of the gradient and the Hessian of $I(\mathbf{Q})$ requires intensive Monte-Carlo simulations.

\section{$D$. The large system approximation of $I(\mathbf{Q})$.}

When $t$ and $r$ converge to $\infty$ while $t / r \rightarrow c, c \in(0, \infty)$, [4] showed that $I(\mathbf{Q})$ can be approximated by $\bar{I}(\mathbf{Q})$ defined by $(7 \mathbf{7}$ at the top of the page, where $\left(\delta_{1}(\mathbf{Q}), \ldots, \delta_{L}(\mathbf{Q})\right)^{T}=\boldsymbol{\delta}(\mathbf{Q})$ and $\left(\tilde{\delta}_{1}(\mathbf{Q}), \ldots, \tilde{\delta}_{L}(\mathbf{Q})\right)^{T}=\tilde{\boldsymbol{\delta}}(\mathbf{Q})$ are the positive solutions of the system of $2 L$ equations:

$$
\left\{\begin{array}{l}
\kappa_{l}=f_{l}(\tilde{\boldsymbol{\kappa}}) \\
\tilde{\kappa}_{l}=\tilde{f}_{l}(\boldsymbol{\kappa}, \mathbf{Q})
\end{array}\right.
$$

with $\boldsymbol{\kappa}=\left(\kappa_{1}, \ldots, \kappa_{L}\right)^{T}$ and $\tilde{\boldsymbol{\kappa}}=\left(\tilde{\kappa}_{1}, \ldots, \tilde{\kappa}_{L}\right)^{T}$, and

$$
\left\{\begin{array}{l}
f_{l}(\tilde{\boldsymbol{\kappa}})=\frac{1}{t} \operatorname{Tr}\left[\mathbf{C}^{(l)} \mathbf{T}(\tilde{\boldsymbol{\kappa}})\right] \\
\tilde{f}_{l}(\boldsymbol{\kappa}, \mathbf{Q})=\frac{1}{t} \operatorname{Tr}\left[\mathbf{Q}^{1 / 2} \tilde{\mathbf{C}}^{(l)} \mathbf{Q}^{1 / 2} \tilde{\mathbf{T}}(\boldsymbol{\kappa}, \mathbf{Q})\right]
\end{array}\right.
$$

where

$$
\left\{\begin{array}{l}
\mathbf{T}^{-1}(\tilde{\boldsymbol{\kappa}})=\sigma^{2}\left(\mathbf{I}+\sum_{j=1}^{L} \tilde{\kappa}_{j} \mathbf{C}^{(j)}\right) \\
\tilde{\mathbf{T}}^{-1}(\boldsymbol{\kappa}, \mathbf{Q})=\sigma^{2}\left(\mathbf{I}+\sum_{j=1}^{L} \kappa_{j} \mathbf{Q}^{1 / 2} \tilde{\mathbf{C}}^{(j)} \mathbf{Q}^{1 / 2}\right)
\end{array}\right.
$$

[4] is based on the replica method, a useful and simple trick whose mathematical relevance is not yet proved in the present context. However, using large random matrix technics similar to those of ([9], [7]), it is possible to prove rigorously that, under mild technical extra assumptions, $\bar{I}(\mathbf{Q})=I(\mathbf{Q})+O\left(\frac{1}{t}\right)$. This point is outside the scope of the present paper.

We also mention that [4] assumed implicitely the existence and the uniqueness of positive solutions of (8) without justification. We therefore precise this important point, and also show that $\left(\delta_{l}(\mathbf{Q})\right)_{l=1, \ldots, L}$ and $\left(\tilde{\delta}_{l}(\mathbf{Q})\right)_{l=1, \ldots, L}$ can be evaluated using a fixed point algorithm.

1) Existence: Using analytic continuation technique and results of [10], it can be shown that the following fixed point algorithm, initialized as follows, converges:

- Initialization: $\delta_{l}^{(0)}>0, \tilde{\delta}_{l}^{(0)}>0, l=1, \ldots, L$.

- Evaluation of the $\delta_{l}^{(n+1)}$ and $\tilde{\delta}_{l}^{(n+1)}$ from $\boldsymbol{\delta}^{(n)}=$ $\left(\delta_{1}^{(n)}, \ldots, \delta_{L}^{(n)}\right)^{T}$ and $\tilde{\boldsymbol{\delta}}^{(n)}=\left(\tilde{\delta}_{1}^{(n)}, \ldots, \tilde{\delta}_{L}^{(n)}\right)^{T}$ :

$$
\left\{\begin{array}{l}
\delta_{l}^{(n+1)}=f_{l}\left(\tilde{\boldsymbol{\delta}}^{(n)}\right) \\
\tilde{\delta}_{l}^{(n+1)}=\tilde{f}_{l}\left(\boldsymbol{\delta}^{(n)}, \mathbf{Q}\right)
\end{array}\right.
$$

Besides, it can be proved that the limit of $\left(\boldsymbol{\delta}^{(n)}, \tilde{\boldsymbol{\delta}}^{(n)}\right)$ when $n \rightarrow \infty$ satisfies equation (8), and that all the entries of this limit are positive. Hence, the convergence of the algorithm yields the existence of a solution to (8).
2) Uniqueness: In order to simplify the notations, we consider in this part the case $\mathbf{Q}=\mathbf{I}$. In order to address the general case, it is sufficient to change matrices $\left(\tilde{\mathbf{C}}^{(l)}\right)_{l=1, \ldots, L}$ into $\left(\mathbf{Q}^{1 / 2} \tilde{\mathbf{C}}^{(l)} \mathbf{Q}^{1 / 2}\right)_{l=1, \ldots, L}$ in what follows. Let $(\boldsymbol{\delta}, \tilde{\tilde{\boldsymbol{\delta}}})$ and $\left(\boldsymbol{\delta}^{\prime}, \tilde{\boldsymbol{\delta}}^{\prime}\right)$ be two solutions of the canonical equation (8). We denote $(\mathbf{T}, \tilde{\mathbf{T}})$ and $\left(\mathbf{T}^{\prime}, \tilde{\mathbf{T}}^{\prime}\right)$ the associated matrices defined by (10). Introducing $\mathbf{e}=\boldsymbol{\delta}-\boldsymbol{\delta}^{\prime}=\left(e_{1}, \ldots, e_{L}\right)^{T}$ we have:

$$
\begin{aligned}
e_{l} & =\frac{1}{t} \operatorname{Tr}\left[\mathbf{C}^{(l)} \mathbf{T}\left(\mathbf{T}^{\prime-1}-\mathbf{T}^{-1}\right) \mathbf{T}^{\prime}\right] \\
& =\frac{\sigma^{2}}{t} \sum_{k=1}^{L}\left(\tilde{\delta}_{k}^{\prime}-\tilde{\delta}_{k}\right) \operatorname{Tr}\left(\mathbf{C}^{(l)} \mathbf{T} \mathbf{C}^{(k)} \mathbf{T}^{\prime}\right)
\end{aligned}
$$

Similarly, with $\tilde{\boldsymbol{e}}=\tilde{\boldsymbol{\delta}}-\tilde{\boldsymbol{\delta}}^{\prime}=\left(\tilde{e}_{1}, \ldots, \tilde{e}_{L}\right)^{T}$,

$$
\tilde{e}_{k}=\frac{\sigma^{2}}{t} \sum_{l=1}^{L}\left(\delta_{l}^{\prime}-\delta_{l}\right) \operatorname{Tr}\left(\tilde{\mathbf{C}}^{(k)} \tilde{\mathbf{T}} \tilde{\mathbf{C}}^{(l)} \tilde{\mathbf{T}}^{\prime}\right)
$$

And (12) and (13) can be written together as

$$
\left[\begin{array}{cc}
\mathbf{I} & \sigma^{2} \mathbf{A}\left(\mathbf{T}, \mathbf{T}^{\prime}\right) \\
\sigma^{2} \tilde{\mathbf{A}}\left(\tilde{\mathbf{T}}, \tilde{\mathbf{T}}^{\prime}\right) & \mathbf{I}
\end{array}\right]\left[\begin{array}{l}
\mathbf{e} \\
\tilde{\mathbf{e}}
\end{array}\right]=\mathbf{0}
$$

with $\mathbf{A}_{k l}\left(\mathbf{T}, \mathbf{T}^{\prime}\right)=\frac{1}{t} \operatorname{Tr}\left(\mathbf{C}^{(k)} \mathbf{T} \mathbf{C}^{(l)} \mathbf{T}^{\prime}\right)$ and $\tilde{\mathbf{A}}_{k l}\left(\tilde{\mathbf{T}}, \tilde{\mathbf{T}}^{\prime}\right)=$ $\frac{1}{t} \operatorname{Tr}\left(\tilde{\mathbf{C}}^{(k)} \tilde{\mathbf{T}} \tilde{\mathbf{C}}^{(l)} \tilde{\mathbf{T}}^{\prime}\right)$. We will now prove that $\rho(\mathbf{M})<1$, with $\mathbf{M}=\sigma^{4} \tilde{\mathbf{A}}\left(\tilde{\mathbf{T}}, \tilde{\mathbf{T}}^{\prime}\right) \mathbf{A}\left(\mathbf{T}, \mathbf{T}^{\prime}\right)$. This will imply that matrix $\mathbf{M}$ is invertible, and thus that $\mathbf{e}=\tilde{\mathbf{e}}=\mathbf{0}$.

$$
\begin{array}{r}
\left|\mathbf{M}_{k l}\right|=\left|\frac{\sigma^{4}}{t^{2}} \sum_{j=1}^{L} \operatorname{Tr}\left(\tilde{\mathbf{C}}^{(k)} \tilde{\mathbf{T}} \tilde{\mathbf{C}}^{(j)} \tilde{\mathbf{T}}^{\prime}\right) \operatorname{Tr}\left(\mathbf{C}^{(j)} \mathbf{T} \mathbf{C}^{(l)} \mathbf{T}^{\prime}\right)\right| \\
\leq \frac{\sigma^{4}}{t^{2}} \sum_{j=1}^{L}\left|\operatorname{Tr}\left(\tilde{\mathbf{C}}^{(k)} \tilde{\mathbf{T}} \tilde{\mathbf{C}}^{(j)} \tilde{\mathbf{T}}^{\prime}\right)\right|\left|\operatorname{Tr}\left(\mathbf{C}^{(j)} \mathbf{T} \mathbf{C}^{(l)} \mathbf{T}^{\prime}\right)\right|
\end{array}
$$

Thanks to the inequality $|\operatorname{Tr}(\mathbf{A B})| \leq \sqrt{\operatorname{Tr}\left(\mathbf{A A}^{\mathbf{H}}\right) \operatorname{Tr}\left(\mathbf{B B}^{\mathbf{H}}\right)}$, we have

$$
\begin{aligned}
& \frac{1}{t}\left|\operatorname{Tr}\left(\tilde{\mathbf{C}}^{(k)} \tilde{\mathbf{T}}^{(j)} \tilde{\mathbf{T}}^{\prime}\right)\right| \leq \sqrt{\tilde{\mathbf{A}}_{k j}(\tilde{\mathbf{T}}, \tilde{\mathbf{T}}) \tilde{\mathbf{A}}_{k j}\left(\tilde{\mathbf{T}}^{\prime}, \tilde{\mathbf{T}}^{\prime}\right)} \\
& \frac{1}{t}\left|\operatorname{Tr}\left(\mathbf{C}^{(j)} \mathbf{T} \mathbf{C}^{(l)} \mathbf{T}^{\prime}\right)\right| \leq \sqrt{\mathbf{A}_{j l}(\mathbf{T}, \mathbf{T}) \mathbf{A}_{j l}\left(\mathbf{T}^{\prime}, \mathbf{T}^{\prime}\right)}
\end{aligned}
$$

Using (16) and (17) in (15) gives

$$
\left|\mathbf{M}_{k l}\right| \leq \sigma^{4} \sum_{j=1}^{L} \sqrt{\tilde{\mathbf{A}}_{k j}(\tilde{\mathbf{T}}) \tilde{\mathbf{A}}_{k j}\left(\tilde{\mathbf{T}}^{\prime}\right) \mathbf{A}_{j l}(\mathbf{T}) \mathbf{A}_{j l}\left(\mathbf{T}^{\prime}\right)}
$$

with $\tilde{\mathbf{A}}(\tilde{\mathbf{T}})=\tilde{\mathbf{A}}(\tilde{\mathbf{T}}, \tilde{\mathbf{T}})$ and $\mathbf{A}(\mathbf{T})=\mathbf{A}(\mathbf{T}, \mathbf{T})$. And, using Cauchy-Schwarz inequality,

$\left|\mathbf{M}_{k l}\right| \leq \sigma^{4} \sqrt{\left(\sum_{j=1}^{L} \tilde{\mathbf{A}}_{k j}(\tilde{\mathbf{T}}) \mathbf{A}_{j l}(\mathbf{T})\right)\left(\sum_{j=1}^{L} \tilde{\mathbf{A}}_{k j}\left(\tilde{\mathbf{T}}^{\prime}\right) \mathbf{A}_{j l}\left(\mathbf{T}^{\prime}\right)\right)}$ 
Hence, we have $\left|\mathbf{M}_{k l}\right| \leq \mathbf{P}_{k l} \forall k, l$, where the matrix $\mathbf{P}$ is defined by $\mathbf{P}_{k l}=\sqrt{\left(\sigma^{4} \tilde{\mathbf{A}}(\tilde{\mathbf{T}}) \mathbf{A}(\mathbf{T})\right)_{k l}} \sqrt{\left(\sigma^{4} \tilde{\mathbf{A}}\left(\tilde{\mathbf{T}}^{\prime}\right) \mathbf{A}\left(\mathbf{T}^{\prime}\right)\right)_{k l}}$. Theorem 8.1.18 of [11] then yields $\rho(\mathbf{M}) \leq \rho(\mathbf{P})$. Besides, Lemma 5.7.9 of [12] used on the definition of $\mathbf{P}$ gives:

$$
\rho(\mathbf{P}) \leq \sqrt{\rho\left(\sigma^{4} \tilde{\mathbf{A}}(\tilde{\mathbf{T}}) \mathbf{A}(\mathbf{T})\right)} \sqrt{\rho\left(\sigma^{4} \tilde{\mathbf{A}}\left(\tilde{\mathbf{T}}^{\prime}\right) \mathbf{A}\left(\mathbf{T}^{\prime}\right)\right)}
$$

We now introduce the following lemma:

Lemma 1: $\rho\left(\sigma^{4} \tilde{\mathbf{A}}(\tilde{\mathbf{T}}) \mathbf{A}(\mathbf{T})\right)<1$

Proof: The $\delta_{l}$ can be written as:

$$
\begin{aligned}
\delta_{l} & =\frac{1}{t} \operatorname{Tr}\left(\mathbf{C}^{(l)} \mathbf{T} \mathbf{T}^{-1} \mathbf{T}\right) \\
& =\frac{\sigma^{2}}{t} \operatorname{Tr}\left(\mathbf{C}^{(l)} \mathbf{T} \mathbf{T}\right)+\frac{\sigma^{2}}{t} \sum_{k=1}^{L} \tilde{\delta_{k}} \operatorname{Tr}\left(\mathbf{C}^{(l)} \mathbf{T} \mathbf{C}^{(k)} \mathbf{T}\right)
\end{aligned}
$$

And $\tilde{\delta}_{l}=\frac{\sigma^{2}}{t} \operatorname{Tr}\left(\tilde{\mathbf{C}}^{(l)} \tilde{\mathbf{T}} \tilde{\mathbf{T}}\right)+\frac{\sigma^{2}}{t} \sum_{k=1}^{L} \delta_{k} \operatorname{Tr}\left(\tilde{\mathbf{C}}^{(l)} \tilde{\mathbf{T}} \tilde{\mathbf{C}}^{(k)} \tilde{\mathbf{T}}\right)$ similarly, thus:

$$
\left[\begin{array}{c}
\boldsymbol{\delta} \\
\tilde{\boldsymbol{\delta}}
\end{array}\right]=\sigma^{2}\left[\begin{array}{cc}
0 & \mathbf{A}(\mathbf{T}) \\
\tilde{\mathbf{A}}(\tilde{\mathbf{T}}) & 0
\end{array}\right]\left[\begin{array}{l}
\boldsymbol{\delta} \\
\tilde{\boldsymbol{\delta}}
\end{array}\right]+\mathbf{v}
$$

This equality is of the form $\mathbf{u}=\mathbf{B u}+\mathbf{v}$, where the entries of $\mathbf{u}$ and $\mathbf{v}$ are positive, and where the entries of $\mathbf{B}$ are nonnegative. A direct application of Corollary 8.1.29 of [11] then implies $\rho(\mathbf{B}) \leq 1-\frac{\min v_{l}}{\max u_{l}}<1$. Noticing that $(\rho(\mathbf{B}))^{2}=$ $\rho\left(\sigma^{4} \tilde{\mathbf{A}}(\tilde{\mathbf{T}}) \mathbf{A}(\mathbf{T})\right)$ ends the proof of Lemma 1

We also have of course $\rho\left(\sigma^{4} \tilde{\mathbf{A}}\left(\tilde{\mathbf{T}}^{\prime}\right) \mathbf{A}\left(\mathbf{T}^{\prime}\right)\right)<1$, so that finally:

$$
\rho(\mathbf{M}) \leq \rho(\mathbf{P})<1
$$

\section{MAXIMIZATION ALGORITHM}

Using the same methods as [13] section IV, the approximation $\bar{I}(\mathbf{Q})$ can be shown to be a strictly concave function over the compact set $\mathcal{C}_{1}$. Therefore it admits a unique argmax that we denote $\overline{\mathbf{Q}}_{*}$. As $\mathcal{C}_{1}$ is convex, it is well known that $\overline{\mathbf{Q}}_{*}$ is characterized by the property

$$
\left\langle\nabla \bar{I}\left(\overline{\mathbf{Q}}_{*}\right), \mathbf{P}-\overline{\mathbf{Q}}_{*}\right\rangle \leq 0
$$

for each matrix $\mathbf{P} \in \mathcal{C}_{1}$, where $\langle\nabla \bar{I}(\mathbf{Q}), \mathbf{P}-\mathbf{Q}\rangle$ represents the limit of $\lambda^{-1}(\bar{I}(\mathbf{Q}+\lambda(\mathbf{P}-\mathbf{Q}))-\bar{I}(\mathbf{Q}))$ when $\lambda \rightarrow 0$, $\lambda>0$. We now consider the function $\mathcal{V}(\mathbf{Q}, \boldsymbol{\kappa}, \tilde{\boldsymbol{\kappa}})$ defined by:

$$
\begin{aligned}
\mathcal{V}(\mathbf{Q}, \boldsymbol{\kappa}, \tilde{\boldsymbol{\kappa}})= & \log |\mathbf{I}+\mathbf{C}(\tilde{\boldsymbol{\kappa}})|+\log |\mathbf{I}+\mathbf{Q} \tilde{\mathbf{C}}(\boldsymbol{\kappa})| \\
& -\sigma^{2} t \sum_{l=1}^{L} \kappa_{l} \tilde{\kappa}_{l}
\end{aligned}
$$

where $\tilde{\mathbf{C}}(\boldsymbol{\kappa})=\sum_{l=1}^{L} \kappa_{l} \tilde{\mathbf{C}}^{(l)}$ and $\mathbf{C}(\tilde{\boldsymbol{\kappa}})=\sum_{l=1}^{L} \tilde{\kappa}_{l} \mathbf{C}^{(l)}$. Note that we have $\mathcal{V}(\mathbf{Q}, \boldsymbol{\delta}(\mathbf{Q}), \tilde{\boldsymbol{\delta}}(\mathbf{Q}))=\bar{I}(\mathbf{Q})$. We have then the following result:

Proposition 1: Denote $\boldsymbol{\delta}_{*}=\boldsymbol{\delta}\left(\mathbf{Q}_{*}\right)$ and $\tilde{\boldsymbol{\delta}}_{*}=\tilde{\boldsymbol{\delta}}\left(\mathbf{Q}_{*}\right)$. Matrix $\mathbf{Q}_{*}$ is the solution of the standard waterfilling problem: maximize over $\mathbf{Q} \in \mathcal{C}_{\mathbf{1}}$ the function $\log \left|\mathbf{I}+\mathbf{Q} \tilde{\mathbf{C}}\left(\boldsymbol{\delta}_{*}\right)\right|$.

Proof: Due to lack of space, only the key points are given. We first remark that maximizing function $\mathbf{Q} \mapsto \log \left|\mathbf{I}+\mathbf{Q} \tilde{\mathbf{C}}\left(\boldsymbol{\delta}_{*}\right)\right|$ is equivalent to maximizing function $\mathbf{Q} \mapsto \mathcal{V}\left(\mathbf{Q}, \boldsymbol{\delta}_{*}, \tilde{\boldsymbol{\delta}}_{*}\right)$ by (19). The proof is then based on the observation that

$$
\begin{aligned}
& \frac{\partial \mathcal{V}}{\partial \kappa_{l}}=-\sigma^{2} t\left(\tilde{f}_{l}(\boldsymbol{\kappa}, \mathbf{Q})-\tilde{\kappa}_{l}\right) \\
& \frac{\partial \mathcal{V}}{\partial \tilde{\kappa}_{l}}=-\sigma^{2} t\left(f_{l}(\tilde{\boldsymbol{\kappa}})-\kappa_{l}\right)
\end{aligned}
$$

are zero at point $(\boldsymbol{\delta}(\mathbf{Q}), \tilde{\boldsymbol{\delta}}(\mathbf{Q}))$. This implies that for each $\mathbf{P} \in$ $\mathcal{C}_{1},\left\langle\nabla \bar{I}\left(\overline{\mathbf{Q}}_{*}\right), \mathbf{P}-\overline{\mathbf{Q}}_{*}\right\rangle$ coincides with $\left\langle\nabla_{\mathbf{Q}} \mathcal{V}\left(\overline{\mathbf{Q}}_{*}, \boldsymbol{\delta}_{*}, \tilde{\boldsymbol{\delta}}_{*}\right), \mathbf{P}-\right.$ $\left.\overline{\mathbf{Q}}_{*}\right\rangle$. As function $\mathbf{Q} \rightarrow \mathcal{V}\left(\mathbf{Q}, \boldsymbol{\delta}_{*}, \tilde{\boldsymbol{\delta}}_{*}\right)$ is strictly concave on $\mathcal{C}_{1}$, (18) implies that its $\operatorname{argmax}$ on $\mathcal{C}_{1}$ coincides with $\overline{\mathbf{Q}}_{*}$.

Proposition 1 shows that the optimum matrix is solution of a waterfilling problem associated to the covariance matrix $\tilde{\mathbf{C}}\left(\boldsymbol{\delta}_{*}\right)$. Although this result provides some insight on the structure of $\overline{\mathbf{Q}}_{*}$, it cannot be used to evaluate it because matrix $\tilde{\mathbf{C}}\left(\boldsymbol{\delta}_{*}\right)$ depends itself of $\overline{\mathbf{Q}}_{*}$. We now introduce an optimization algorithm of $\bar{I}(\mathbf{Q})$; the iterative scheme is the following:

- Initialization: $\mathbf{Q}_{0}=\mathbf{I}$

- Evaluation of $\mathbf{Q}_{k}$ from $\mathbf{Q}_{k-1}:\left(\boldsymbol{\delta}^{(k)}, \tilde{\boldsymbol{\delta}}^{(k)}\right)$ is defined as the unique solution of (8) in which $\mathbf{Q}=\mathbf{Q}_{k-1}$. Then $\mathbf{Q}_{k}$ is defined as the maximum of function $\mathbf{Q} \mapsto$ $\log \left|\mathbf{I}+\mathbf{Q} \tilde{\mathbf{C}}\left(\boldsymbol{\delta}^{(k)}\right)\right|$ on $\mathcal{C}_{1}$.

We now establish a result which shows that if the algorithm converges, then it converges towards $\overline{\mathbf{Q}}_{*}$.

Proposition 2: Assume that

$$
\lim _{k \rightarrow \infty} \boldsymbol{\delta}^{(k)}-\boldsymbol{\delta}^{(k-1)}=\lim _{k \rightarrow \infty} \tilde{\boldsymbol{\delta}}^{(k)}-\tilde{\boldsymbol{\delta}}^{(k-1)}=0
$$

Then, the algorithm converges torwards matrix $\overline{\mathbf{Q}}_{*}$.

Proof: Due to the lack of space, we just outline the proof which is similar to the proof of Proposition 6 of [7]. As $\mathcal{C}_{1}$ is compact, we have just to verify that each convergent subsequence $\left(\mathbf{Q}_{\psi(k)}\right)_{k \in \mathbb{N}}$ extracted from $\left(\mathbf{Q}_{k}\right)_{k \in \mathbb{N}}$ converges towards $\overline{\mathbf{Q}}_{*}$. For this, we denote by $\overline{\mathbf{Q}}_{\psi, *}$ the limit of the above subsequence, and prove that this matrix verifies property (18). We first remark that sequences $\boldsymbol{\delta}^{\psi(k)+1}$ and $\tilde{\boldsymbol{\delta}}^{\psi(k)+1}$ converge towards vectors denoted $\boldsymbol{\delta}^{\psi, *}$ and $\tilde{\boldsymbol{\delta}}^{\psi, *}$ respectively. Moreover, $\left(\boldsymbol{\delta}^{\psi, *}, \tilde{\boldsymbol{\delta}}^{\psi, *}\right)$ is solution of system (8) in which matrix $\mathbf{Q}$ coincides with $\overline{\mathbf{Q}}_{\psi, *}$. Therefore, using relations (20) and (21) as in the proof of Proposition 1, we obtain that $\left\langle\nabla \bar{I}\left(\overline{\mathbf{Q}}_{\psi, *}\right), \mathbf{P}-\right.$ $\left.\overline{\mathbf{Q}}_{\psi, *}\right\rangle$ coincides with $\left\langle\nabla_{\mathbf{Q}} \mathcal{V}\left(\overline{\mathbf{Q}}_{\psi, *}, \boldsymbol{\delta}_{\psi, *}, \tilde{\boldsymbol{\delta}}_{\psi, *}\right), \mathbf{P}-\overline{\mathbf{Q}}_{\psi, *}\right\rangle$. It remains to show that this term is negative for each $\mathbf{P}$ to complete the proof. For this, we use that $\mathbf{Q}_{\psi(k)}$ is the argmax over $\mathcal{C}_{1}$ of function $\mathbf{Q} \rightarrow \mathcal{V}\left(\mathbf{Q}, \boldsymbol{\delta}^{\psi(k)}, \tilde{\boldsymbol{\delta}}^{\psi(k)}\right)$. Therefore,

$$
\left\langle\nabla_{\mathbf{Q}} \mathcal{V}\left(\mathbf{Q}_{\psi(k)}, \boldsymbol{\delta}_{\psi(k)}, \tilde{\boldsymbol{\delta}}_{\psi(k)}\right), \mathbf{P}-\mathbf{Q}_{\psi(k)}\right\rangle \leq 0
$$

By (22), sequences $\left(\boldsymbol{\delta}_{\psi(k)}\right)_{k \geq 0}$ and $\left(\tilde{\boldsymbol{\delta}}_{\psi(k)}\right)_{k \geq 0}$ converge towards $\boldsymbol{\delta}^{\psi, *}$ and $\tilde{\boldsymbol{\delta}}^{\psi, *}$ respectively. Taking the limit of 23) when $k \rightarrow \infty$ shows that $\left\langle\nabla_{\mathbf{Q}} \mathcal{V}\left(\overline{\mathbf{Q}}_{\psi, *}, \boldsymbol{\delta}_{\psi, *}, \tilde{\boldsymbol{\delta}}_{\psi, *}\right), \mathbf{P}-\right.$ $\left.\overline{\mathbf{Q}}_{\psi, *}\right\rangle \leq 0$ as required. 
TABLE I

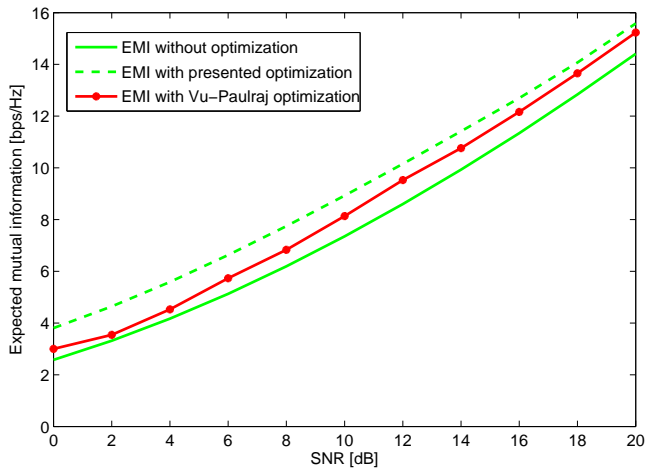

Fig. 1. Comparison with Vu-Paulraj algorithm

To conclude, if the algorithm is convergent, that is, if the sequence of $\left(\mathbf{Q}_{k}\right)_{k \in \mathbb{N}}$ converges towards a certain matrix, then the $\delta_{l}^{(k)}=\delta_{l}\left(\mathbf{Q}_{k-1}\right)$ and the $\tilde{\delta}_{l}^{(k)}=\tilde{\delta}_{l}\left(\mathbf{Q}_{k-1}\right)$ converge as well when $k \rightarrow \infty$. (22) is verified, hence, if the algorithm is convergent it converges towards $\overline{\mathbf{Q}}_{*}$. Although the convergence of the algorithm has not been proved, this result is encouraging and suggests that the algorithm is reliable. In particular, in all the conducted simulations the algorithm was converging. In any case, condition (22) can be easily checked. If it is not satisfied, it is possible to modify the initial point $\mathbf{Q}_{0}$ as many times as needed to ensure the convergence.

\section{Numerical Results}

We provide here some simulations results to visualize the impact of the transmit correlation optimization in a realistic context. We use the propagation model introduced in [3], in which each path corresponds to a scatterer cluster characterized by a mean angle of departure and an angle spread.

In the featured simulations, we consider a frequency selective MIMO system with $t=r=4$, a carrier frequency of $2 \mathrm{GHz}$, a number of paths $L=5$. The paths share the same power, and their mean departure angles and angles spreads are given in Table \$ (in radians). In Figure 1 we have represented the true EMI $I(\mathbf{I})$ (i.e. without optimization), and the optimized EMI $I\left(\overline{\mathbf{Q}}_{*}\right)$ (i.e. with an input covariance matrix maximizing the approximation). The EMI are evaluated by Monte-Carlo simulations, with $10^{5}$ channel realizations. The EMI optimized with Vu-Paulraj algorithm [5] is also represented for comparison. We fixed to 10 the number of iterations in [5].

Figure 1 shows that maximizing $\bar{I}(\mathbf{Q})$ over the input covariance leads to significant improvement for $I(\mathbf{Q})$. Our approach provides the same results as Vu-Paulraj's algorithm at high SNR, and performs even better elsewhere. Vu-Paulraj's approach is penalized by the barrier method if the optimal input covariance is close to be singular, which is here the case if the SNR is not high enough. Moreover our algorithm is computationally much more efficient: in Vu-Paulraj's algorithm the evaluation of the gradient and of the Hessian of $I(\mathbf{Q})$ needs heavy Monte-Carlo simulations ( $10^{4}$ trials were used).
PATHS ANGULAR PARAMETERS (in radians)

\begin{tabular}{|c|c|c|c|c|c|}
\cline { 2 - 6 } \multicolumn{1}{c|}{} & $l=1$ & $l=2$ & $l=3$ & $l=4$ & $l=5$ \\
\hline mean departure angle & 6.15 & 3.52 & 4.04 & 2.58 & 2.66 \\
\hline departure angle spread & 0.06 & 0.09 & 0.05 & 0.05 & 0.03 \\
\hline mean arrival angle & 4.85 & 3.48 & 1.71 & 5.31 & 0.06 \\
\hline arrival angle spread & 0.06 & 0.08 & 0.05 & 0.02 & 0.11 \\
\hline
\end{tabular}

TABLE II

AVERAGE EXECUTION TIME (in seconds)

\begin{tabular}{|c|c|c|c|}
\cline { 2 - 4 } \multicolumn{1}{c|}{} & $L=3$ & $L=4$ & $L=5$ \\
\hline Vu-Paulraj & 903 & 1245 & 1649 \\
\hline New algorithm & $7,0.10^{-3}$ & $7,4.10^{-3}$ & $8,3.10^{-3}$ \\
\hline
\end{tabular}

Table II gives for both algorithms the average execution time in seconds to obtain the input covariance matrix, on a $3.16 \mathrm{GHz}$ Intel Xeon CPU with 8GB of RAM, for a number of paths $L=3, L=4$ and $L=5$.

\section{CONCLUSION}

In this paper we have addressed the evaluation of the capacity achieving covariance matrices of frequency selective MIMO channels. We have proposed to optimize a large system approximation of the EMI, and have introduced an attractive iterative algorithm.

\section{REFERENCES}

[1] A. Goldsmith, S. Jafar, N. Jindal, and S. Vishwanath, "Capacity limits of MIMO channels," IEEE J. Select. Areas Commun., vol. 21, no. 5, pp. 684-702, 2003.

[2] D. Hoesli, Y. Kim, and A. Lapidoth, "Monotonicity results for coherent MIMO Rician channels," IEEE Trans. Inform. Theory, vol. 51, no. 12, pp. 4334-4339, 2005.

[3] H. Bolckei, D. Gesbert, and A. Paulraj, "On the capacity of OFDMbased spatial multiplexing systems," IEEE Trans. Commun., vol. 50, no. 2, pp. 225-234, 2002.

[4] A. Moustakas and S. Simon, "On the outage capacity of correlated multiple-path MIMO channels," IEEE Trans. Inform. Theory, vol. 53, no. 11, p. 3887, 2007.

[5] M. Vu and A. Paulraj, "Capacity optimization for Rician correlated MIMO wireless channels," in Proc. Asilomar Conference, 2005, pp. $133-138$.

[6] C. Wen, P. Ting, and J. Chen, "Asymptotic analysis of MIMO wireless systems with spatial correlation at the receiver," IEEE Trans. Commun., vol. 54, no. 2, pp. 349-363, 2006.

[7] J. Dumont, W. Hachem, S. Lasaulce, P. Loubaton, and J. Najim, "On the capacity achieving covariance matrix for Rician MIMO channels: an asymptotic approach," IEEE Trans. Inform. Theory, vol. 56, no. 3, pp. 1048-1069, 2010.

[8] C. Artigue, P. Loubaton, and B. Mouhouche, "On the ergodic capacity of frequency selective MIMO systems equipped with MMSE receivers: An asymptotic approach," in Proc. Globecom, 2008.

[9] W. Hachem, O. Khorunzhiy, P. Loubaton, J. Najim, and L. Pastur, "A new approach for capacity analysis of large dimensional multi-antenna channels," IEEE Trans. Inform. Theory, vol. 54, no. 9, 2008.

[10] W. Hachem, P. Loubaton, and J. Najim, "Deterministic equivalents for certain functionals of large random matrices," Annals of Applied Probability, vol. 17, no. 3, pp. 875-930, 2007.

[11] R. Horn and C. Johnson, Matrix analysis. Cambridge Univ Pr, 1990.

[12] - Topics in matrix analysis. Cambridge Univ Pr, 1994.

[13] J. Dumont, W. Hachem, S. Lasaulce, P. Loubaton, and J. Najim, "On the capacity achieving covariance matrix for Rician MIMO channels: an asymptotic approach," 2007. [Online]. Available: http://arxiv.org/abs/0710.4051 